\title{
PAR-CLIP data indicate that Nrd1-Nab3-dependent transcription termination regulates expression of hundreds of protein coding genes in yeast
}

Shaun Webb², Ralph D Hector ${ }^{1}$, Grzegorz Kudla ${ }^{3}$ and Sander Granneman ${ }^{1,2^{*}}$

\begin{abstract}
Background: Nrd1 and Nab3 are essential sequence-specific yeast RNA binding proteins that function as a heterodimer in the processing and degradation of diverse classes of RNAs. These proteins also regulate several mRNA coding genes; however, it remains unclear exactly what percentage of the mRNA component of the transcriptome these proteins control. To address this question, we used the PyCRAC software package developed in our laboratory to analyze CRAC and PAR-CLIP data for Nrd1-Nab3-RNA interactions.

Results: We generated high-resolution maps of Nrd1-Nab3-RNA interactions, from which we have uncovered hundreds of new Nrd1-Nab3 mRNA targets, representing between 20 and 30\% of protein-coding transcripts. Although Nrd1 and Nab3 showed a preference for binding near $5^{\prime}$ ends of relatively short transcripts, they bound transcripts throughout coding sequences and 3' UTRs. Moreover, our data for Nrd1-Nab3 binding to 3' UTRs was consistent with a role for these proteins in the termination of transcription. Our data also support a tight integration of Nrd1-Nab3 with the nutrient response pathway. Finally, we provide experimental evidence for some of our predictions, using northern blot and RT-PCR assays.
\end{abstract}

Conclusions: Collectively, our data support the notion that Nrd1 and Nab3 function is tightly integrated with the nutrient response and indicate a role for these proteins in the regulation of many mRNA coding genes. Further, we provide evidence to support the hypothesis that Nrd1-Nab3 represents a failsafe termination mechanism in instances of readthrough transcription.

\section{Background}

RNA binding proteins play crucial roles in the synthesis, processing and degradation of RNA in a cell. To better understand the function of RNA binding proteins, it is important to identify their RNA substrates and the sites of interaction. This helps to better predict their function and lead to the design of more focused functional analyses. Only recently, the development of cross-linking and immunoprecipitation (CLIP) and related techniques has made it possible to identify direct protein-RNA interactions in vivo at a very high resolution [1-5]. To isolate direct protein-RNA interactions, cells are UV irradiated to forge covalent bonds between the protein of interest

\footnotetext{
* Correspondence: sgrannem@staffmail.ed.ac.uk

${ }^{1}$ Centre for Synthetic and Systems Biology (SynthSys), Mayfield Road, Kings

Buildings, Waddington Building office 3.06, Edinburgh EH9 3JD, UK

${ }^{2}$ Wellcome Trust Centre for Cell Biology, University of Edinburgh, Edinburgh EH9 3JR, UK

Full list of author information is available at the end of the article
}

and bound RNAs. The target protein is subsequently affinity purified under stringent conditions, and UV crosslinked RNAs are partially digested, ligated to adapters, RT-PCR amplified and sequenced. CLIP methods are becoming increasingly popular and produce valuable data. The number of papers describing the technique seems to double every year and it is now being applied in a wide range of organisms. The method is also under constant development: the individual-nucleotide resolution CLIP (iCLIP) approach has improved the accuracy of mapping cross-linking sites $[2,4]$, and incorporating photoactivatable nucleotides in RNA can enhance the UV cross-linking efficiency [1]. We have recently developed a stringent affinity-tag-based CLIP protocol (cross-linking and cDNA analysis (CRAC)) that can provide a higher specificity [5], and the tag-based approach is becoming more widely adopted $[4,6]$. The combination of CLIP with high-throughput sequencing (for example, HITS-CLIP) 
has markedly increased the sensitivity of the methodology and provided an unparalleled capability to identify protein-RNA interactions transcriptome-wide [3,5,7]. This approach is producing a lot of extremely valuable high-throughput sequencing data. Fortunately, many bioinformatics tools are now becoming available tailored to tackle the large CRAC/CLIP datasets [8-11]. We have recently developed a python package, dubbed pyCRAC, that conveniently combines many popular CLIP/CRAC analysis methods in an easy to use package.

Nrd1 and Nab3 are essential sequence-specific yeast RNA binding proteins that function as a heterodimer in processing and degradation of diverse classes of RNAs [12-19]. Transcription termination of RNA polymerase (Pol) II transcripts generally involves mRNA cleavage and addition of long polyA tails (cleavage and polyadenylation (CPF) pathway), which label the RNA ready for nuclear export (reviewed in [20]). By contrast, transcripts terminated by Nrd1-Nab3 generally contain short polyA tails and are substrates for the nuclear RNA degradation machinery [21,22]. This activity is also important for small nucleolar RNA (snoRNA) maturation and degradation of cryptic unstable transcripts (CUTs) and stable unannotated transcripts (SUTs) [12,23-26]. Nrd1 and Nab3 direct transcription termination of nascent transcripts by interacting with the highly conserved carboxy-terminal domain (CTD) of RNA polymerase II. Because this interaction requires phosphorylation at serine 5 in the CTD, $\mathrm{Nrd} 1$ and Nab3 are believed to primarily operate on promoter proximal regions where serine 5 phosphorylation levels are high $[27,28]$.

Recent high-throughput studies have indicated Nrd1 and Nab3 frequently UV cross-link to mRNAs $[6,24,29]$ and thousands of mRNA coding genes harbor Nrd1 and Nab3 binding sequences (see below). However, thus far a relatively small number of mRNAs have been reported to be targeted by Nrd1 and Nab3 [25,30-33]. Indeed, it is not clear exactly what percentage of the mRNA transcriptome these proteins control. To address this question, we reanalyzed CRAC and PAR-CLIP data using the pyCRAC software package. We generated high-resolution maps of Nrd1-Nab3-RNA interactions, focusing on the presence of known RNA binding motifs in the sequencing data. We also confirmed some of our predictions experimentally. Our analyses revealed that Nrd1-Nab3 bound between 20 to $30 \%$ of protein-coding transcripts, several hundred of which had binding sites in untranslated regions (UTRs). Although Nrd1 and Nab3 showed a preference for binding near $5^{\prime}$ ends of relatively short transcripts, they bound transcripts throughout coding sequences and 3' UTRs. Our data suggest that Nrd1-Nab3 can terminate transcription of a long approximately $5 \mathrm{~kb}$ transcript by binding 3' UTRs and we speculate that the fate of many mRNAs is dictated by kinetic competition between
Nrd1-Nab3 and the CPF termination pathways. Statistical analyses revealed that Nrd1 and Nab3 targets are significantly enriched for enzymes and permeases involved in nucleotide/amino acid synthesis and uptake, and for proteins involved in mitochondrial organization. Collectively, our data support the notion that Nrd1 and Nab3 function is tightly integrated with the nutrient response [30] and indicate a role for these proteins in the regulation of many mRNA coding genes.

\section{Results and discussion}

\section{Identification of Nrd1-Nab3 binding sites in PAR-CLIP data}

Previous genetic and biochemical studies have identified a number of short Nrd1 and Nab3 RNA binding motifs (UCUU and CUUG in Nab3; UGUA and GUAG in Nrd1) $[6,15,16,18,24,29]$. Not surprisingly, almost every single mRNA coding gene in the yeast genome contains at least one copy of these motifs and could therefore be Nrd1 and Nab3 targets (see below). To get an impression of how many mRNAs are actually targeted by Nrd1 and $\mathrm{Nab3}$ in yeast, we analyzed data from Nrd1 and Nab3 CLIP/CRAC experiments using the pyCRAC software package [34].

Two high-throughput protein-RNA cross-linking studies on Nrd1 and Nab3 in yeast have recently been described using PAR-CLIP [6,29] and the CRAC method [24]. Both studies produced very similar results and indicated that Nrd1 and Nab3 target RNAs generated by all three RNA polymerases. Here we focus on the PAR-CLIP data, as the number of uniquely mapped reads in these datasets was higher and allowed identification of a greater number of targets (data not shown). Figure 1 provides a schematic overview of how the read data were processed. All identical read sequences were removed and only reads with unique chromosomal mapping positions were considered (Figure 1A,B). Negative control CLIP experiments often do not generate sufficient material for generating high quality cDNA libraries for sequencing. Because no control PAR-CLIP samples were available, we calculated the minimum read coverage (or 'height') required to obtain a false discovery rate (FDR) of less than 0.01 for each annotated feature in the genome. Read contigs were generated from those regions with coverage higher than, or equal to, the minimum height (Figure 1C). We reasoned that this approach would reduce noise and sequence representation biases introduced by highly expressed genes. A potential drawback of this approach is that genes with high read coverage (such as tRNAs) are less likely to contain significantly enriched regions, leading to an underestimation of the number of binding sites in these genes.

We next searched for overrepresented sequences in Nrd1 and Nab3 read contigs (Figure 1E). Consistent with recently published work $[24,29]$, previously identified Nrd1-Nab3 motifs were highly over-represented 


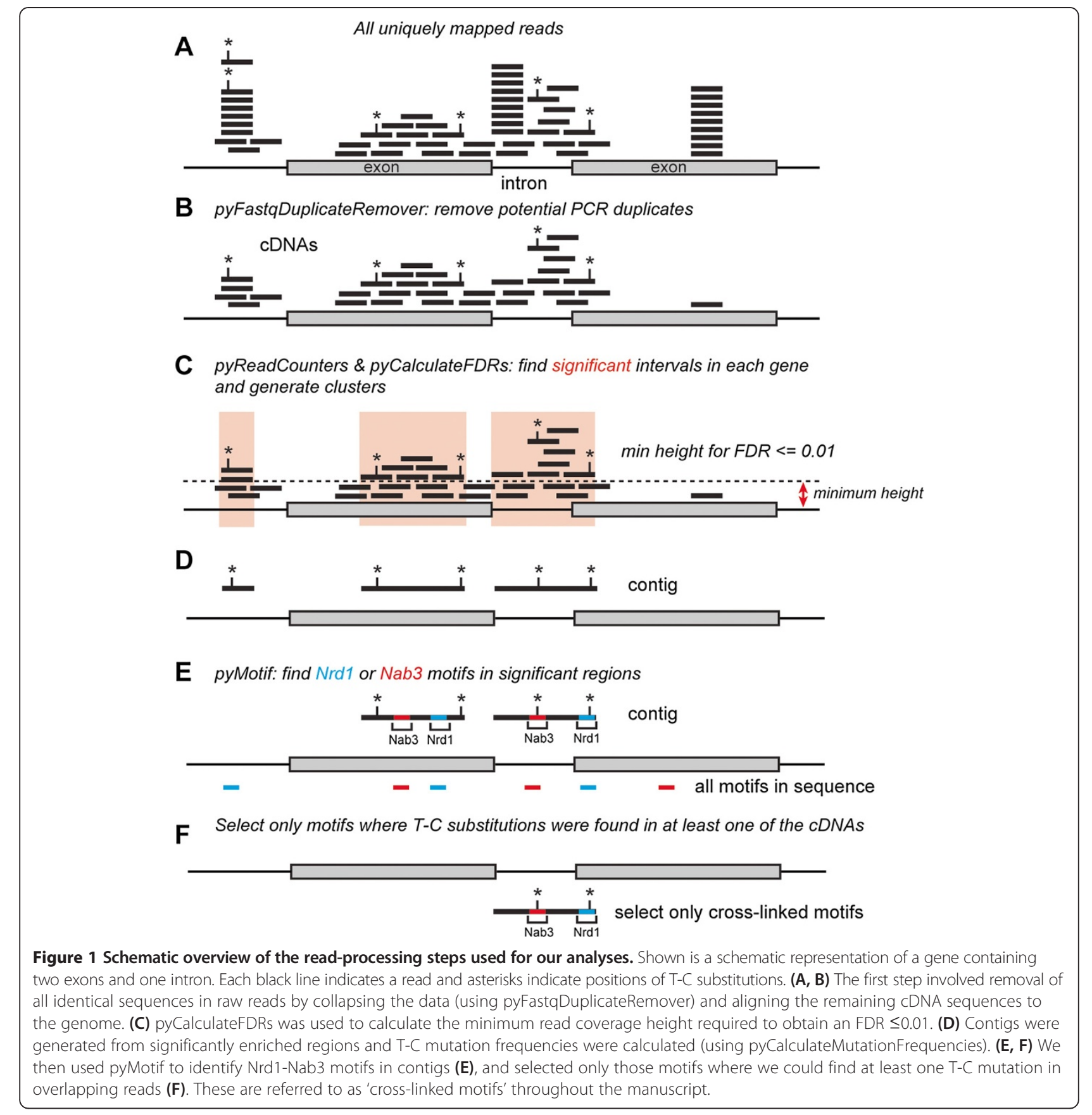

(Table S1 in Additional file 1). Additionally, the recently described AU-rich Nrd1 motifs (UGUAA and UGUAAA) $[29,35]$ were among the top scoring 5- and 6-mers, respectively. Because UV-induced cross-linking sites in PAR-CLIP data are often highlighted by T-C substitutions [1], we reasoned we could obtain higher confidence binding sites by focusing on motif sequences isolated from contigs that contained a $\mathrm{T}-\mathrm{C}$ substitution in at least one overlapping read (Figure 1D-F). All T-C substitutions in reads were weighted equally and included as mutations in contigs (Figure 1D). Additional file 2 shows that T-C mutations in contigs generated from the Nrd1 PAR-CLIP data were clearly enriched over Nrd1 motifs, confirming that Nrd1 has a strong preference for cross-linking to these sites $[6,24,29]$. Sequence contigs generated from the Nab3 data sets had high T-C mutation frequencies (Figure S1B in Additional file 2) and only a modest enrichment could be seen downstream of Nab3 motifs. This result is in contrast with recent analyses performed on Nab3 CRAC data, where cross-linking sites were mainly detected within UCUU and CUUG sequences (Figure S1C in Additional file 2) [24]. This discrepancy could be, in 

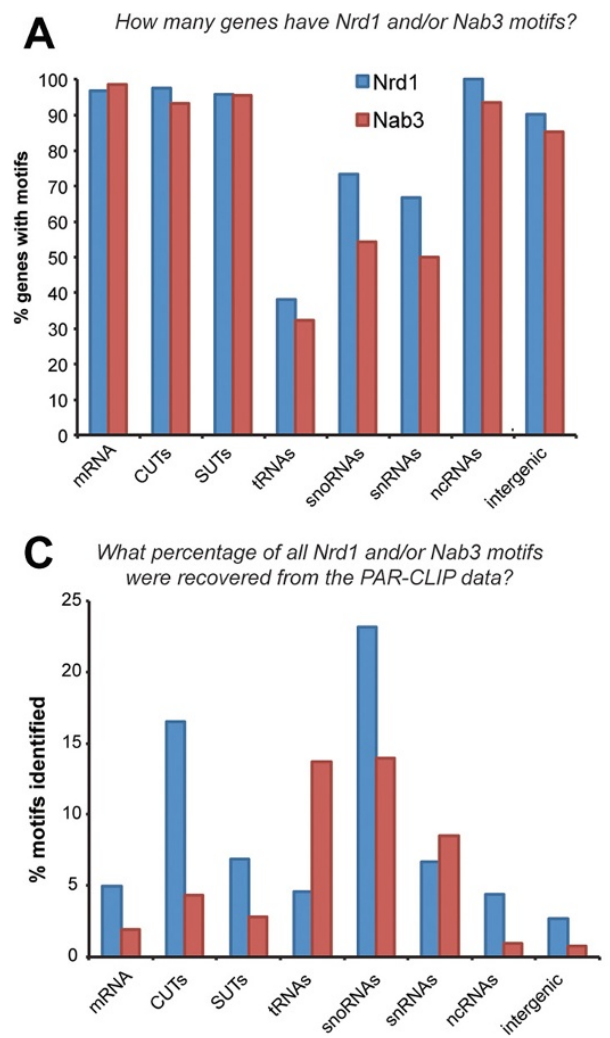

Figure 2 Comparison of predicted and identified binding sites. (A) Overview of the percentage ( $y$-axis) of genes in genomic features ( $x$-axis) that contain Nrd1 (blue) or Nab3 (red) motifs in their sequence. (B) The percentage of genomic features that contained cross-linked Nrd1 or Nab3 motifs. (C) The percentage of all Nrd1 and Nab3 motifs in gene/feature sequences found in the PAR-CLIP data analyses. (D) The distribution of cross-linked motifs over UTR and exon sequences. ncRNA, non-coding RNA; snRNA, small nuclear RNA.

part, the result of noise in the Nab3 PAR-CLIP data, as other short sequences were more highly enriched in Nab3 contigs than the previously reported Nab3 binding sites (Table S1 in Additional file 1). To reduce noise, we only selected Nab3 motifs containing T-C substitutions from contigs (Figure 1F), hereafter referred to as 'cross-linked motifs'. Overall, our motif analyses are in excellent agreement with previously published work.

\section{At least a quarter of the mRNAs are Nrd1-Nab3 targets}

Figure 2A provides an overview of the percentage of genes in the genome that contain Nrd1 (UGUA, GUAG) and Nab3 (UCUU, CUUG) motifs. The vast majority of motifs were found in protein coding genes and cryptic Pol II transcripts such as CUTs and SUTs. Although generally fewer motifs were present in short non-coding RNA genes (tRNAs, small nuclear RNAs (snRNAs) and snoRNAs; Figure 2A), a high percentage of these motifs contained T-C substitutions in the PAR-CLIP data (Figure 2C). Many Nrd1 and Nab3 motifs are located in snoRNA flanking regions, which were not included in our analyses. Therefore, the number provided here is an underestimation of the total snoRNA targets. Strikingly,

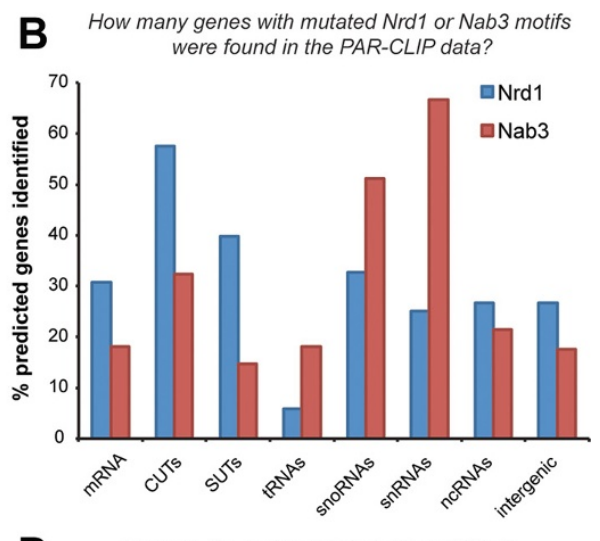

D How are the motifs distributed in mRNAs?

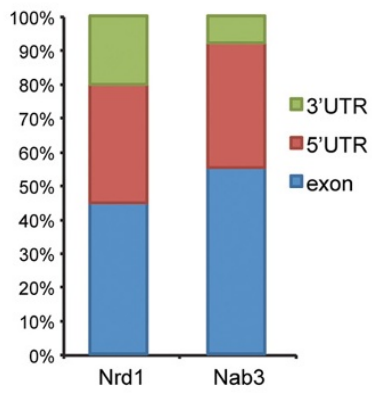

the PAR-CLIP analyses showed that Nrd1 and Nab3 crosslinked to 20 to $30 \%$ of the approximately 6,300 mRNA transcripts analyzed (Figure $2 \mathrm{~B}$ ), although only a relatively small fraction of all motifs present in the genomic sequence contained $\mathrm{T}-\mathrm{C}$ substitutions (less than 5\%; Figure $2 \mathrm{C}$ ). Around $50 \%$ of the cross-linked motifs mapped to untranslated regions, with a preference for 5' UTRs (Figure 2D). Consistent with recently published data, our analyses identified the telomerase RNA (TLC1) as a Nrd1-Nab3 target $[29,36]$. Other non-coding RNA targets included the RNase P RNA (RPR1), the signal recognition particle RNA (SCR1) and ICR1. Collectively, our analyses uncovered over a thousand mRNAs that could be regulated by Nrd1 and Nab3.

\section{Nrd1 and Nab3 preferentially bind to $5^{\prime}$ ends of a subset of mRNA transcripts}

To refine our analyses, we generated genome-wide coverage plots for cross-linked Nrd1 and Nab3 motifs and compared them to the distribution of the motifs present in the genome (Figure 3A). UTR and transcript lengths were normalized by dividing the sequences in an equal number of bins. For each bin we estimated the Nab3/Nrd1 binding 


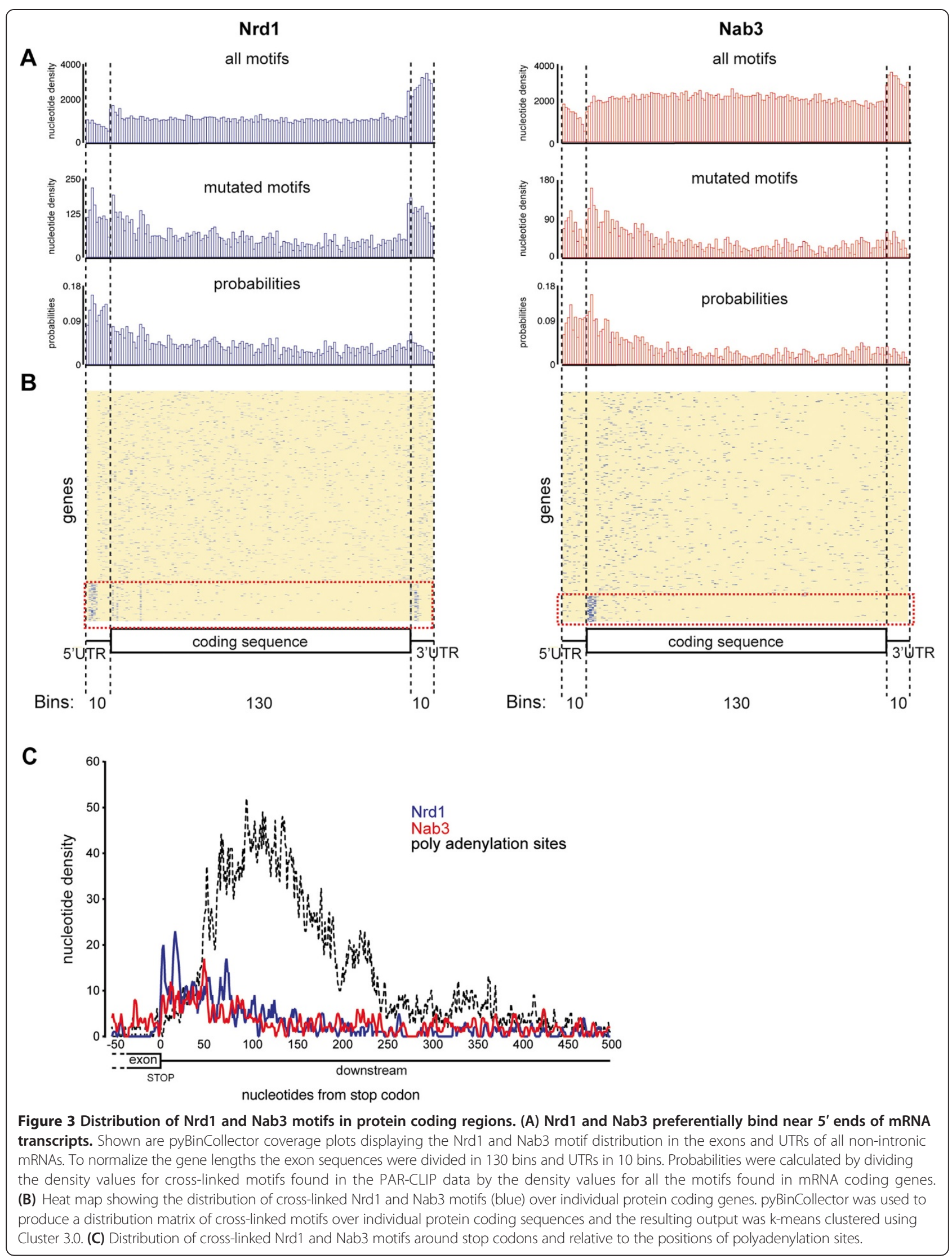


probability by dividing the number of cross-linked motifs by the total number of motifs in that bin. To evaluate the quality of the coverage plots, we generated heat maps displaying the distribution of Nrd1 and Nab3 motifs in individual protein coding genes (Figures $3 \mathrm{~B}$ and 4 ).

Both Nrd1 and Nab3 are co-transcriptionally recruited to the Pol II CTD. Chromatin immunoprecipitation (ChIP) experiments have indicated a preference for Nrd1-Nab3 binding near the $5^{\prime}$ ends of protein coding genes [27,28,37]. Binding of Nrd1 and Nab3 near the $5^{\prime}$ end of transcripts can lead to premature transcription termination and it was proposed that this was a regulatory mechanism for downregulating mRNA levels. Indeed, transcriptome-wide, the probability of finding cross-linked motifs was higher near the $5^{\prime}$ end of protein coding genes (Figure 3A). However, the heat maps in Figure 3B show that the distribution of cross-linked motifs over mRNAs varied considerably, and indicated that a relatively small number of genes mostly contributed to the signal near 5 ' ends. K-means clustering of the pyBinCollector data revealed 308 transcripts where cross-linked Nrd1 and/or Nab3 motifs concentrated near $5^{\prime}$ ends (highlighted by a red-dotted line in Figures 3B and 4), primarily downstream of the transcription start site (TSS) (Figure 4). This group included previously described Nrd1-Nab3 targets, such as PCF11, URA8 and NRD1 (Figures 4 and 5A) [6,25,29] and therefore may represent a group of genes that are regulated by Nrd1-Nab3-dependent premature transcription termination. Notably, this group also included numerous other genes required for mRNA 3' end formation as well as genes encoding turnover and export factors (Figures 4 and 5B; PAP2/TRF4, PTI1, REF2, DHH1, NAB2, TEX1, PTI1, NOT5). We speculate that Nrd1 and Nab3 can regulate mRNA metabolism at many levels.

Gene Ontology term analyses on this list of transcripts also revealed a significant enrichment of enzymes with oxidoreductase activity (almost $10 \%$; $P$-value $<0.02$ ) and genes involved in cellular transport activities such as nitrogen compounds $(8.8 \%$; $P$-value $=0.0069)$. These included genes involved in ergosterol biosynthesis (Figure $5 \mathrm{C}$; ERG24, ERG3 and ERG4), nucleoporins (KAP114, KAP108/ SXM1, KAP121/PSE1, KAP142/MSN5), several nucleoside and amino acid permeases (FUR4, MEP3, MMP1, DIP5, $C A N 1, F C Y 2, B A P 3$; Figure $5 \mathrm{D})$ and various other transporters (TPO1, TPO3, TAT1, YCF1).

Regulation of many genes involved in nucleotide biosynthesis is dictated by nucleotide availability and involves selection of alternative TSSs (IMD2, URA2, URA8 and $A D E 12)$ [42-45]. When nucleotide levels are sufficient, transcription starts at upstream TSSs and the elongating polymerase reads through Nrd1-Nab3 binding sites. When Nrd1-Nab3 bind these transcripts they are targeted for degradation. Indeed, several of the transcripts that originate from alternative TSSs have been annotated as CUTs. For a number of genes we could also detect cross-linked motifs upstream of the TSSs. Interestingly, cryptic transcription (XUTs and/or CUTs) was detected just upstream of AIM44, CDC47/MCM7, DIP5, ERG24, EMI2, FCY2, FRE1, GPM2, IRA2, MIG2, MYO1, TIR2, TEX1, YOR352W and YGR269W [38,39] (red colored gene names in Figure 4), hinting that these genes could also be regulated via alternative start site selection.

Collectively, these data are consistent with a role for Nrd1 and Nab3 in the nutrient response pathway [30] and we speculate that Nrd1-Nab3-dependent premature termination is a more widely used mechanism for regulating mRNA levels than was previously anticipated [25].

\section{Nrd1 and Nab3 bind 3' UTRs of several hundred mRNAs}

Nrd1 and Nab3 have been shown to regulate expression of mRNA transcripts by binding 3' UTRs. It was proposed that in cases where the polymerase fails to terminate at conventional polyadenylation sites, Nrd1 and Nab3 binding to 3' UTRs could act as a transcription termination 'fail-safe' mechanism [32]. From our data we predict that this is likely a widely used mechanism to prevent Pol II from transcribing beyond normal transcription termination sites.

We identified a total of 373 transcripts (approximately $6 \%$ of all protein coding genes analyzed) where crosslinked Nrd1 and/or Nab3 motifs mapped to 3' UTRs (Table S2 in Additional file 1). Two examples are shown in Figure 5B,E. We identified several cross-linked Nrd1 and Nab3 motifs downstream of the MSN1 and NAB2 coding sequences. We speculate that these are examples of 'fail-safe' termination, where Nrd1 and Nab3 prevent read-through transcription into neighboring genes located on the same (TRF4) or opposite strand (RPS2). This arrangement of termination sites is reminiscent of the region downstream of $R P L 9 B$ (Figure $5 F$ ), where the CPF and Nrd1-Nab3 termination machineries act in competition [33]. Cross-linked Nrd1 motifs also appeared enriched near the 3 ' ends of protein coding genes (Figure 5A,B). The Nrd1 GUAG and GUAA motifs contain stop codons and we found that indeed a fraction of the cross-linked Nrd1 motifs recovered from the PAR-CLIP data overlapped with stop-codons (Figure 5C).

A role for Nrd1-Nab3-dependent 3' end processing of mRNA has also been described: the TIS11/CTH2 mRNA is generated from approximately 1,800-nucleotide, 3' extended precursors and binding of Nrd1 and Nab3 to $3^{\prime}$ UTRs recruits the exosome that is responsible for trimming the extended RNAs [31]. Our analysis identified 6 cross-linked Nrd1-Nab3 motifs within this 1,800 CTH2 nucleotide region (Figure 6A) and we could find several other examples of genes with a similar organization of binding sites. One striking example was TRA1, a component of the SAGA and NuA4 histone acetyltransferase 


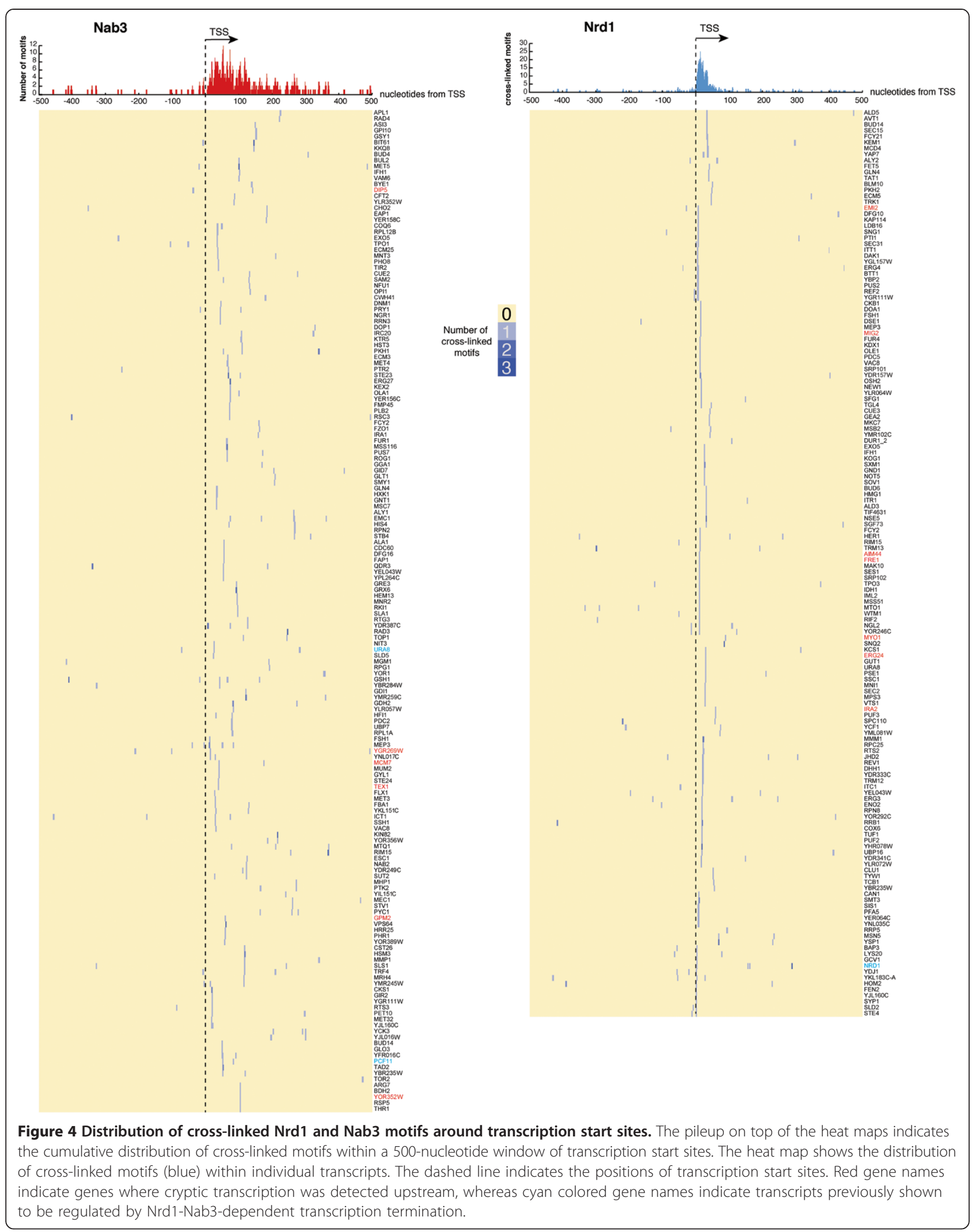




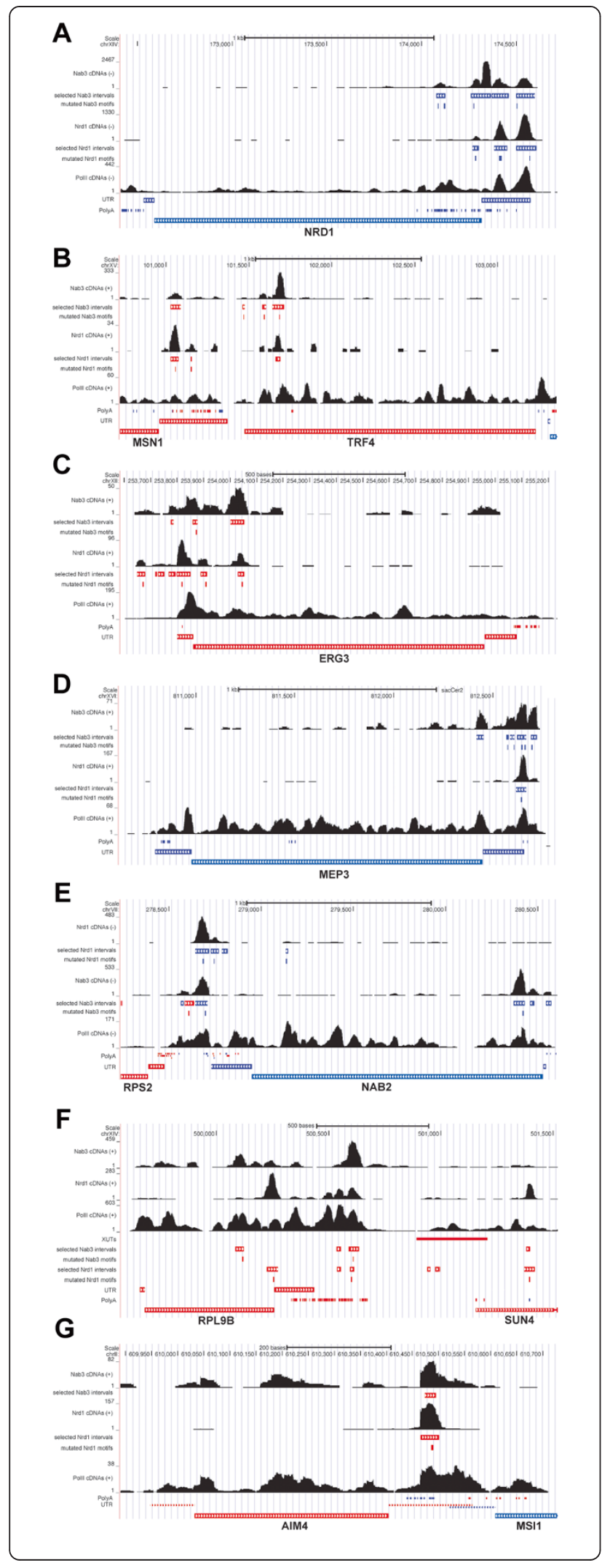

Figure $5 \mathrm{Nrd} 1$ and $\mathrm{Nab} 3$ binding to a selected number of protein-coding transcripts. (A-G) Shown are UCSC genome browser images for a number of genes predicted to be regulated by Nrd1-Nab3. Coverage of unique cDNAs from Nrd1, Nab3 and Pol II (Rpb2) PAR-CLIP data $[6,29]$ on Watson (+) and Crick (-) strands is shown as black histograms. Locations of cross-linked Nrd1-Nab3 motifs (this work), annotated Xrn1-sensitive unstable transcripts (XUTs), polyadenylation sites and UTRs [22,38-41] are included as rectangles. Genomic features located on the Watson (+) strand are indicated in red, whereas features on the Crick (-) strand are indicated in blue. 'Selected intervals' indicate genomic regions with a read coverage FDR $\leq 0.01$. These were used for pyMotif analyses.

complex (Figure 6B). Several Nrd1-Nab3 peaks and four cross-linked Nrd1 motifs were identified downstream of the TRA1 coding sequence. Notably, the downstream regions of CTH2 and TRA1 overlap with transcripts annotated as 'anti-sense regulatory non-coding RNAs' (Xrn1-sensitive unstable transcripts (XUTs)) [46], raising the question of whether these XUTs are products of readthrough transcription.

\section{Nrd1-Nab3 and mitochondrion organization}

The Corden laboratory recently demonstrated a role for Nrd1 in mitochondrial DNA maintenance [30]. An $n r d 1$ 102 temperature-sensitive mutant showed a higher mitochondrial DNA content and was synthetically lethal with an AIM37 deletion, a gene involved in mitochondrial inheritance $[30,47]$. Remarkably, a statistically significant fraction of the cross-linked Nrd1 and Nab3 motifs located in 3' UTRs mapped to genes involved in mitochondrial organization and maintenance (37 genes, $P$-value 0.011 ). These include those encoding the mitochondrial DNA binding protein (ILV5), the nuclear pore associated protein (AIM4; Figure 5G), a large number of proteins that localize to the mitochondrial inner membrane (COX16, COX17, FCJ1, TIM12, TIM14/PAM18, TIM54, YLH47, YTA12, CYC2, COA3, OXA1) and several mitochondrial ribosomal proteins (NAM9, MRP13, MRPL3, MRPL21, MRPL22 and MRPL38). Notably, cells lacking AIM4 show similar defects in mitochondrial biogenesis as an aim37 strain [47].

Collectively, the data suggest that Nrd1 and Nab3 play an important role in mitochondrial function and development.

\section{$\mathrm{Nab3}$ is required for fail-safe termination of the convergent} HHT1 and IPP1 genes

To substantiate our results we analyzed expression levels of several genes that we predicted were regulated by Nrd1-Nab3 (Figure 7A). For these analyses we used strains in which the Nrd1 and Nab3 genes were placed under the control of a galactose inducible/glucose repressible promoter (GAL/GLU; Figure 7B), allowing us to deplete these proteins by growing the cells in glucosecontaining medium using well established conditions 


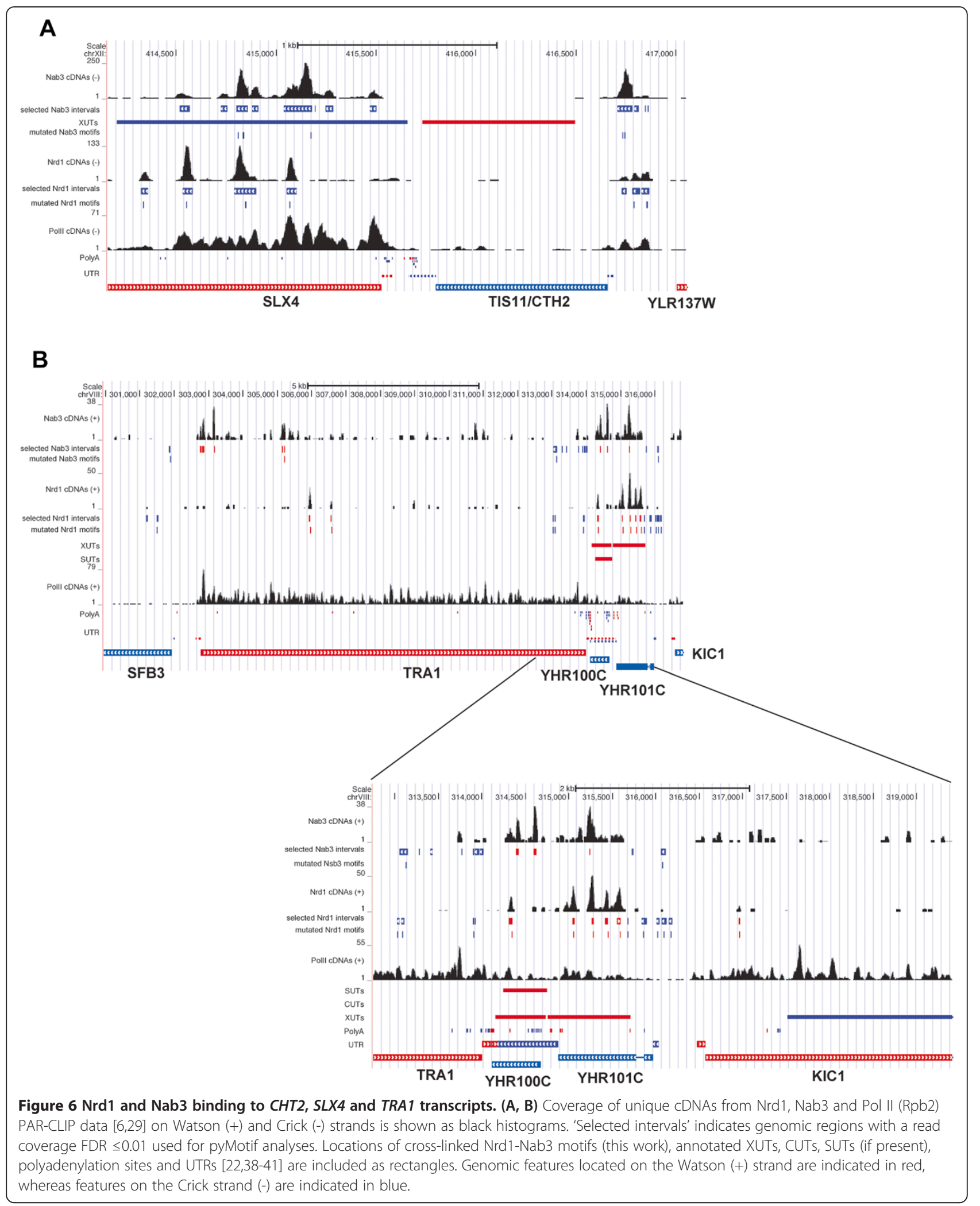




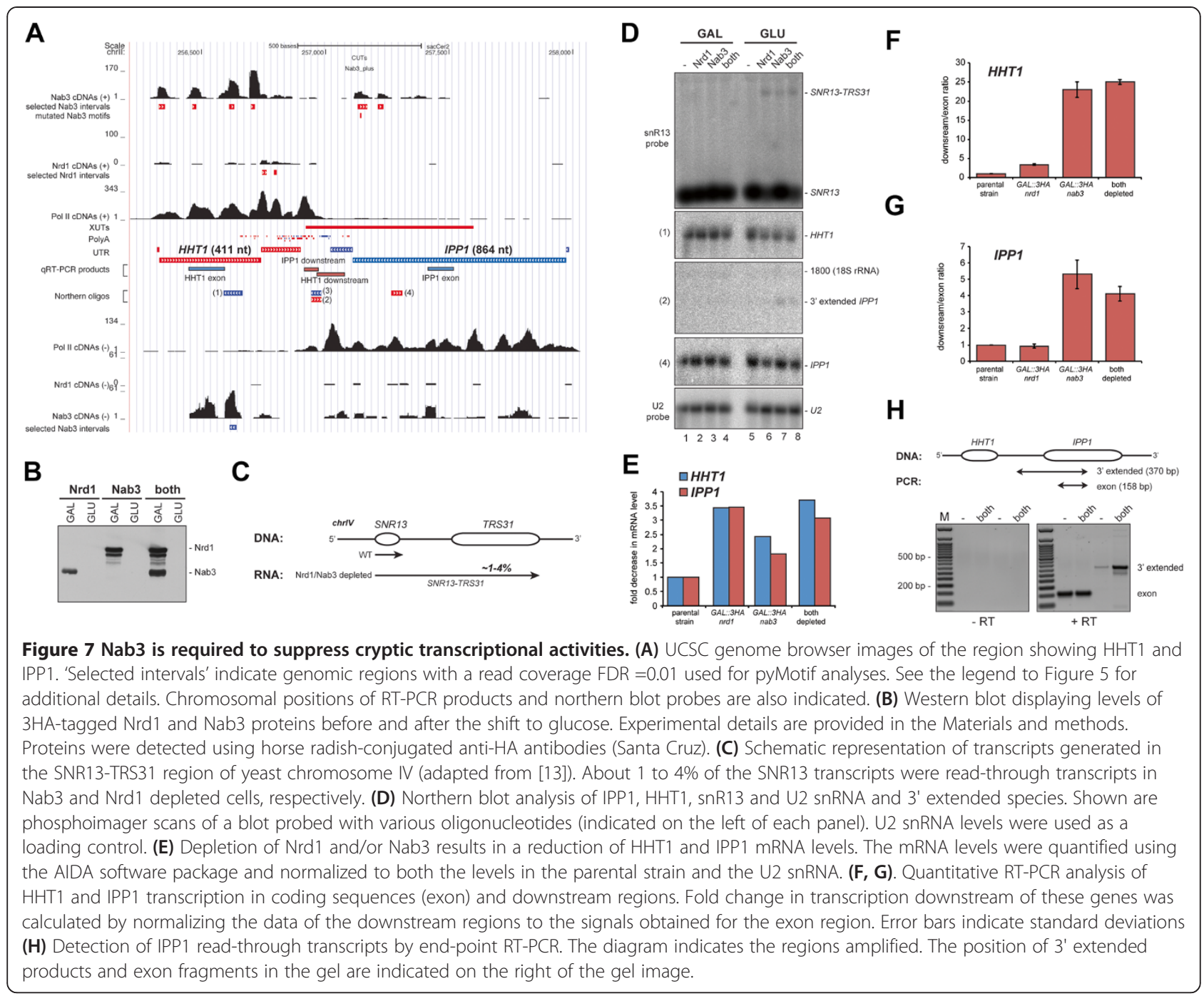

[24]. Transcript levels were analyzed by northern blotting and/or RT-PCR (endpoint and quantitative; Figures 7 and 8). Consistent with previous work [13], northern blot analyses showed that depletion of Nrd1 and/or Nab3 resulted in read-through transcription beyond the SNR13 gene through the TSR31 gene (Figure 7C,D). Under the depletion conditions used, between 1\% (Nrd1depleted) and 3.5\% (Nab3-depleted) of the SNR13 RNAs were read-through transcripts (Figure 7C).

The convergent HHT1 and IPP1 genes came to our attention because we identified a cross-linked Nab3 motif that mapped to a XUT located directly downstream of the HHT1 gene (Figure 7A). XUTs can silence expression of neighboring sense genes by modulating their chromatin state [46]; therefore, this XUT could play a role in regulating IPP1 expression. In addition, substantial Nab3 crosslinking was also observed to anti-sense HHT1 transcripts (Figure 7A). We predicted that Nab3 was required to suppress multiple cryptic transcriptional activities in this region.

Quantification of the northern data shown in Figure 7D revealed a two- to four-fold reduction in HHT1 and IPP1 mRNA levels in the absence of Nrd1 and/or Nab3 (Figure 7E). These results indicate a role for $\mathrm{Nrd} 1$ and Nab3 in regulating mRNA levels of these genes.

We were unable to detect the XUT by northern blotting, presumably because it is rapidly degraded by RNA surveillance machineries (using oligo 3; Figure 7A; data not shown). However, quantitative RT-PCR (qRT-PCR) results showed a staggering approximately 25 -fold increase in XUT levels in the absence of Nab3 (Figure 7F), clearly demonstrating a role for Nab3 in suppressing the expression of this XUT. The Pol II PAR-CLIP data revealed transcription downstream of the IPP1 polyadenylation signals (Figure 7A), indicating that a fraction of polymerases did not terminate at these sites. Depletion of Nab3 resulted 


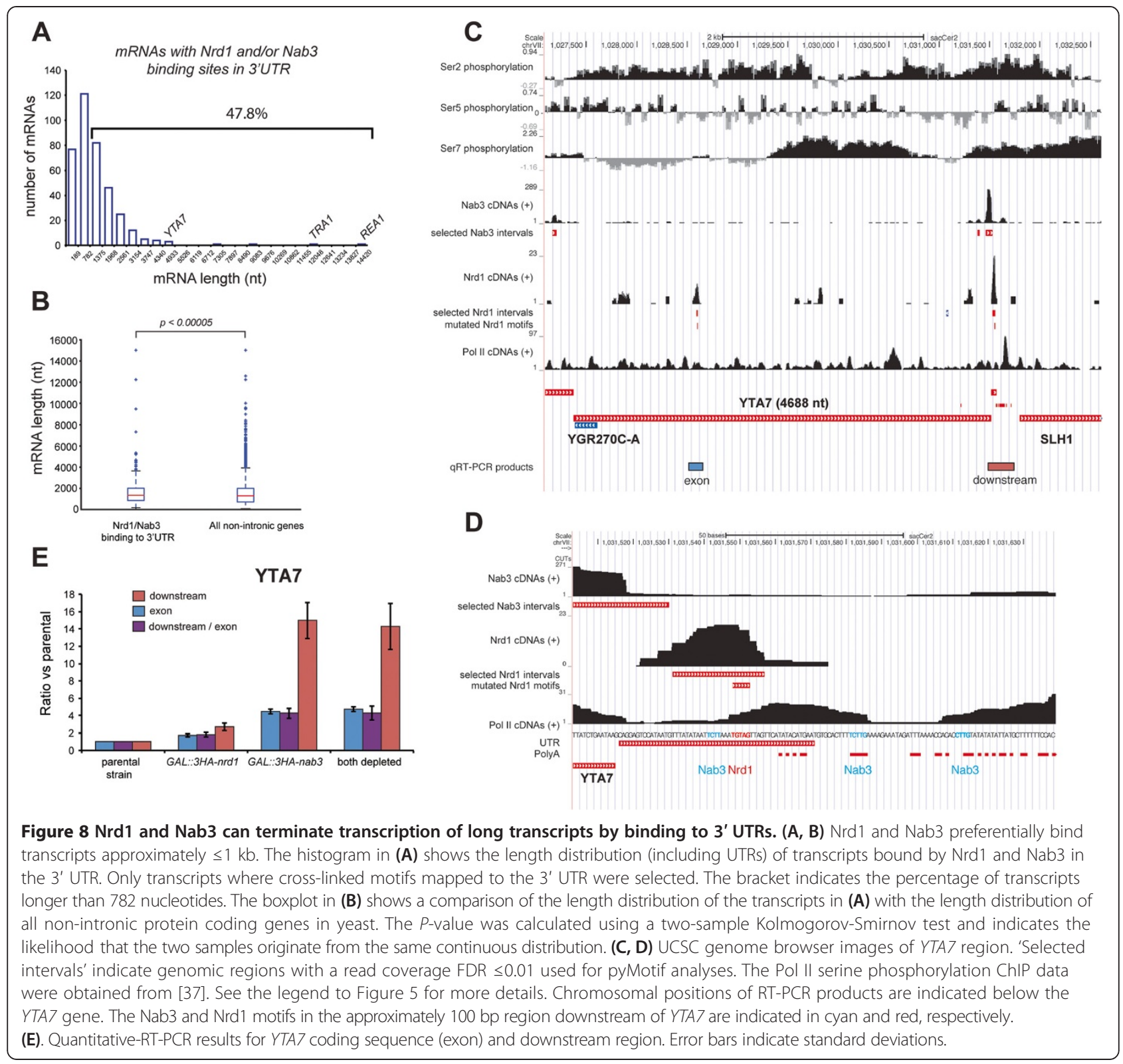

in an approximately six-fold increase in transcription downstream of the annotated IPP1 polyadenylation sites (Figure 7G) and low levels of IPP1 read-through transcripts could be detected by northern blotting and endpoint RT-PCR (Figure 7D,H). We conclude that here Nab3 functions as a 'fail-safe' terminator by preventing the polymerase from transcribing beyond the IPP1 polyadenylation sites into the HHT1 gene. Consistent with the low level of Nrd1 cross-linking in this region, Nrd1 depletion only modestly increased the XUT levels and no significant increase in read-through transcription of IPP1 could be detected (Figure 7A,D,G). These data indicate a role for Nab3 in fail-safe termination of IPP1 and suppressing XUT expression, which may interfere with transcription of genes on the opposite strand.

\section{Nrd1-Nab3-dependent transcription termination of long mRNA transcripts}

The level of serine 5 phosphorylated CTD gradually decreases during transcription of coding sequences, and it has been shown that Nrd1-dependent transcription termination becomes less efficient once approximately 900 nucleotides have been transcribed $[27,28]$. Almost half of the transcripts bound by both Nrd1 and Nab3 in the 3' UTR were longer than approximately 800 nucleotides (Figure 8A). However, compared to the length distribution 
of all the analyzed protein coding genes, both proteins did preferentially cross-link to transcripts smaller than $1 \mathrm{~kb}$ (Figure 8B). To determine whether Nrd1-Nab3 can terminate transcripts longer than $1 \mathrm{~kb}$, we monitored transcription of the approximately $4.7 \mathrm{~kb} Y T A 7$ gene in Nrd1-Nab3 depleted cells. The YTA7 transcript was selected because significant cross-linking of Nrd1 and Nab3 was detected mainly in the 3' UTR. Notably, contrary to the IPP1 transcript, Nrd1-Nab3 cross-linked primarily upstream of polyadenylation sites, indicating that Nrd1-Nab3 termination could precede CPF-dependent termination (Figure 8C,D). The strength of Nrd1-Nab3-dependent transcription termination depends on at least three factors: (1) the number of clustered Nrd1-Nab3 motifs in a sequence, (2) the organization of the binding sites and (3) the presence of AU-rich sequences surrounding the binding sites [16,35]. Three Nab3 motifs were located within 70 nucleotides of the cross-linked Nrd1 motif in the 3' UTR of YTA7, which were surrounded by AU-rich polyadenylation sequences (Figure $8 \mathrm{D}$ ). This indicates that this region has the required signals for Nrd1-Nab3directed transcription termination. To address this, we performed qRT-PCR with oligonucleotides that amplify sequences downstream of the YTA7 3' UTR. We also measured YTA7 mRNA levels by using oligonucleotides that amplify a fragment of the YTA7 exon (Figure 8E). The results show that depletion of Nrd1 and/or Nab3 led to an increase in transcription downstream of the YTA7 3' UTR (Figure 8E), indicating read through. However, we can not exclude the possibility that these transcripts represent different isoforms of the same gene [48]. As with IPP1, depletion of Nab3 had by far the strongest effect (Figure 8E). Strikingly, we could also detect two- to four-fold increase in YTA7 mRNA levels in the absence of these proteins. This suggests that, by default, a significant fraction of YTA7 is degraded via the Nrd1-Nab3 termination pathway.

Genome-wide ChIP data had indicated that Nrd1 binding correlated with serine 7 phosphorylation of the Pol II CTD, whereas recruitment of factors required for conventional CPF pathway correlated with serine 2 phosphorylation [37]. Both serine 7 and serine 2 phosphorylation peaked in the 3' UTR of YTA7 (Figure 8C) [37], indicating that both the Nrd1-Nab3 and CPF termination pathways are active in this region. This organization of termination signals is frequently found in cryptic transcripts (CUTs) [35], many of which are downregulated via the Nrd1Nab3 pathway. It appears that a similar mechanism is used to regulate YTA7 mRNA levels and our bioinformatics analyses suggest that several hundred genes could be regulated in this way; we are currently investigating this in more detail. Transcriptome-wide, the Nrd1-Nab3 UV cross-linking profiles change when cells are starved of glucose [6]. It is conceivable, therefore, that the expression levels of these genes are dictated by the nutrient availability.

\section{Conclusions}

We have presented a comprehensive analysis of Nrd1 and Nab3 PAR-CLIP datasets using the pyCRAC tool suite. We have uncovered more than a thousand potential Nrd1Nab3 mRNA targets and our data indicate that Nrd1-Nab3 play an important role in the nutrient response and mitochondrial function. We have also provided valuable biological insights into regulation of mRNA transcription by the Nrd1-Nab3 termination pathway. Our data support a role for Nab3 in 'fail-safe' termination and regulation of XUT expression. Moreover, we demonstrate that Nrd1-Nab3 can terminate transcription of long transcripts and downregulate mRNA levels by binding to 3' UTRs. We speculate that at least several hundreds of genes are regulated in this way. We are confident that the analyses presented here will be a useful resource for groups working on transcription termination.

\section{Materials and methods pyCRAC software}

The data described here were generated using pyCRAC version 1.1, which can be downloaded from [34]. The Galaxy version is available on the Galaxy tool-shed at [49] and requires pyCRAC to be installed in the /usr/ local/bin/ directory.

\section{Sequence and feature files}

All Gene Transfer Format (GTF) annotation and genomic sequence files were obtained from ENSEMBL. Genomic coordinates for annotated CUTs, SUTs, TSSs, polyadenylation sites and UTRs were obtained from the Saccharomyces Genome Database (SGD) [22,38-41]. To visualize the data in the UCSC genome browser the pyGTF2bed and pyGTF2bedGraph tools were used to convert pyCRAC GTF output files to a UCSC compatible bed format.

\section{Raw data processing and reference sequence alignment}

Nrd1, Nab3 and Pol II (Rpb2) PAR-CLIP datasets were downloaded from the Gene Expression Omnibus (GEO) database (GSM791764, Nrd1; GDM791765, Rpb2; GSM791767; Nab3). The fastx_toolkit [50] was used to remove low quality reads, read artifacts and adapter sequences from fastq files. Duplicate reads were removed using the pyCRAC pyFastqDuplicateRemover tool. Reads were mapped to the 2008 S. cerevisiae genome (version EF2.59) using novoalign version 2.07 [51] and only cDNAs that mapped to a single genomic location were considered.

\section{Counting overlap with genomic features}

PyReadCounters was used to calculate overlap between aligned cDNAs and yeast genomic features. To simplify 
the analyses, we excluded intron-containing mRNAs. UTR coordinates were obtained from the Saccharomyces Genome Database (SGD) [40,52]. The yeast genome version EF2.59 genomic feature file (2008; ENSEMBL) was used for all the analyses described here.

\section{Calculation of motif false discovery rates}

The pyCalculateFDRs script uses a modified version of a FDR algorithm implemented in Pyicos [9]. For a detailed explanation of how the algorithm works, please see the pyCRAC documentation. Reads overlapping a gene or genomic feature were randomly distributed a hundred times over the gene sequence and FDRs were calculated by dividing the probability of finding a region in the PAR-CLIP data with the same coverage by the probability of finding the same coverage in the gene in the randomized data. We only selected regions with an FDR $\leq 0.01$.

\section{Motif analyses}

The motif analyses were performed using the pyMotif tool from the pyCRAC suite. To indicate overrepresentation of a k-mer sequence in the experimental data, pyMotif calculates Z-scores for each k-mer, defined as the number of standard deviations by which an actual $\mathrm{k}$-mer count minus the k-mer count from random data exceeds zero. K-mers were extracted from contigs that mapped sense or anti-sense to yeast genomic features. Repetitive sequences in reads or clusters were only counted once to remove biases towards homopolymeric sequences. Bedtools was used to extract motifs that overlap with genomic features such as exons and UTRs and plots were generated using Gnuplot. The EMBOSS tool fuzznuc was used to extract genomic coordinates for all possible Nrd1 and Nab3 binding and the output files were converted to the GTF format.

\section{Generation of genome-wide coverage plots}

PyBinCollector was used to generate the coverage plots. To normalize the gene lengths, the tool divided the gene sequences over an equal number of bins. For each read, cluster (and their mutations), it calculated the number of nucleotides that map to each bin (referred to as nucleotide densities). To plot the distribution of T-C mutations over the 4 nucleotide Nrd1-Nab3 RNA binding motifs, we added 50 nucleotides up- and downstream of genomic coordinates for each identified motif, and divided these into 104 bins, yielding one nucleotide per bin and the motif start at bin 51 . We then calculated the number of T-C substitutions that map to each bin and divided the number by the total number of Ts in each bin, yielding T-C substitution percentages. To plot the distribution of cross-linked motifs around TSSs, we included 500 nucleotides up- and downstream of the start sites and divided these into 1,001 bins, yielding one nucleotide per bin. To generate the heat maps shown in Figures 3 and 4, we used the -outputall flag in pyBinCollector. The resulting data were K-means clustered using Cluster 3.0 [53]. Heat maps were generated using TreeView [54].

\section{Western and northern blot analyses}

Western blot analyses and genetic depletion of Nrd1-Nab3 using $G A L:: 3 H A$ strains were performed as previously described [24]. Briefly, cells were grown in YPGalRaf ( $2 \%$ galactose, $2 \%$ raffinose) to an OD600 of approximately 0.5 and shifted to YPD medium (2\% glucose) for 9 (GAL::3HAnrd1/GAL::3HA-nab3), 10 (GAL::3HA-nrd1) or 12 hours (GAL::3HA-nab3). Total RNA extraction was performed as previously described [55]. Northern blotting analyses were performed using ULTRAhyb-Oligo according to the manufacturer's procedures (Ambion Austin, TX, USA). Oligonucleotides used in this study are listed in Table S3 in Additional file 1. Nrd1 and Nab3 proteins were detected using horse radish-conjugated anti-HA antibodies (Santa Cruz, Dallas, TX, USA; 1:5,000)

\section{RT-PCR analyses}

The oligonucleotide primers used for the RT-PCR analyses are listed in Table S3 in Additional file 1. Total RNA was treated with DNase I (Ambion) according to the manufacturer's instructions. For the qRT-PCR analyses, RNA was reverse-transcribed and amplified using qScript One-Step SYBR Green qRT-PCR (Quanta Bioscience, Gaithersburg, MD, USA), performed on a Roche LightCycler 480 according to the manufacturer's instructions (Roche, Burgess Hill, UK). Each reaction contained $50 \mathrm{ng}$ template RNA and $250 \mathrm{nM}$ gene-specific primers. Thermal cycling conditions were composed of $50^{\circ} \mathrm{C}$ for 5 minutes, $95^{\circ} \mathrm{C}$ for 2 minutes, followed by 40 cycles of $95^{\circ} \mathrm{C}$ for $3 \mathrm{~s}, 60^{\circ} \mathrm{C}$ for $30 \mathrm{~s}$. Appropriate no-RT and no-template controls were included in each assay, and a dissociation analysis was performed to test assay specificity. Relative quantification in gene expression was calculated using the Roche LightCycler 480 Software. YTA7 levels were normalized to the levels of the PPM2 transcript (NM_00118395) where no significant cross-linking of Nrd1 and Nab3 was detected. For the end-point RT-PCR reactions, 100 ng of total RNA was reverse transcribed using Superscript III at $50^{\circ} \mathrm{C}$ according to the manufacturers instructions (Invitrogen, Paisley, UK) and $2 \mu \mathrm{M}$ of IPP1 reverse primer. The PCR included 200 $\mathrm{nM}$ of forward primers. Thermal cycling conditions were 35 cycles of: $95^{\circ} \mathrm{C}$ for $30 \mathrm{~s}, 60^{\circ} \mathrm{C}$ for $30 \mathrm{~s}$ and then $72^{\circ} \mathrm{C}$ for 1 minute.

\section{Additional files}

Additional file 1: Table S1. PyMotif identified previously described Nrd1-Nab3 RNA binding motifs. Shown are overrepresented 4- to 6-mers (k-mers) and corresponding Z-scores that were isolated from the Nrd1 $(11,964)$ 
and Nab3 $(18,222)$ intervals with an FDR value of $<0.01$. Previously identified Nrd1 and Nab3 motifs are highlighted in red and blue, respectively. Table S2. Transcripts that contained cross-linked Nrd1-Nab3 motifs in 3' UTRs. Table S3. Oligonucleotides used in this study.

Additional file 2: Figure S1. Mutations are enriched in and around Nrd1 and Nab3 RNA binding motifs. (A, B) Analysis of T-C mutations found in the PAR-CLIP data near Nab3 and Nrd1 RNA binding motifs. (C) Analysis of deletions found in the Nab3 CRAC data around the Nab3 motifs [24]. pyBinCollector was used to calculate the coverage of T-C mutations or deletions in read contigs (see main text) within a 50-nucleotide window over Nab3 (CUUG, UCUU) (B, C) and Nrd1 (UGUA, GUAG) (A) motifs identified in the genome. To calculate T-C conversion percentages, the number of T-C substitutions was divided by the total number of Ts at each position. The asterisks indicate the positions in the motif where most frequently T-C substitutions were found in the Nrd1-Nab3 motifs.

\section{Abbreviations}

ChIP: Chromatin immunoprecipitation; CLIP: Cross-linking and immunoprecipitation; CPF: Cleavage and polyadenylation; CRAC: Cross-linking and CDNA analysis; CTD: Carboxy-terminal domain; CUT: Cryptic unstable transcript; FDR: False discovery rate; GTF: Gene Transfer Format; PCR: Polymerase chain reaction; Pol: RNA polymerase; qRT-PCR: Quantitative RT-PCR; snoRNA: Small nucleolar RNA; snRNA: Small nuclear RNA; SUT: Stable unannotated transcript; TSS: Transcription start site; UTR: Untranslated region; XUT: Xrn1-sensitive unstable transcript.

\section{Competing interest}

The authors declare that they have no competing interests.

\section{Authors' contributions}

SW implemented pyCRAC in Galaxy, RDH performed the qRT-PCR experiments, GK aided in the development pyBarcodeFilter and pyMotif. SG conceived and performed the experimental and computational procedures and developed the pyCRAC tools. SW, RDH, GK and SG wrote the paper. All authors read and approved the final manuscript for publication.

\section{Acknowledgements}

We would like to thank Jai Tree, Louise McGibbon, Rebecca Holmes, Alex Tuck and many beta-testers from outside our University for testing the pyCRAC tools and help with debugging the scripts. We are very grateful to Steve West, Lidia Vasilieva and Jeffry Corden for critically reading the manuscript and David Tollervey for his support and advice. We would like to thank Alastair Kerr, Stuart Aitken and Christos Josephides for helpful suggestions on the implementation of algorithms for statistical analyses. This work was supported by Wellcome Trust Research and Career Development Grants (097383 to GK, 091549 to SG), the Medical Research Council (GK) and the Wellcome Trust Centre for Cell Biology core grant (092076).

\section{Author details}

'Centre for Synthetic and Systems Biology (SynthSys), Mayfield Road, Kings Buildings, Waddington Building office 3.06, Edinburgh EH9 3JD, UK. ${ }^{2}$ Wellcome Trust Centre for Cell Biology, University of Edinburgh, Edinburgh EH9 3JR, UK. ${ }^{3}$ MRC Human Genetics Unit, University of Edinburgh, Western General Hospital, Crewe Road, Edinburgh EH4 2XU, UK.

Received: 8 October 2013 Accepted: 7 January 2014 Published: 7 January 2014

\section{References}

1. Hafner M, Landthaler M, Burger L, Khorshid M, Hausser J, Berninger P, Rothballer A, Ascano M, Jungkamp A-C, Munschauer M, Ulrich A, Wardle GS, Dewell S, Zavolan M, Tuschl T: Transcriptome-wide identification of RNA-binding protein and microRNA target sites by PAR-CLIP. Cell 2010, 141:129-141.

2. König J, Zarnack K, Rot G, Curk T, Kayikci M, Zupan B, Turner DJ, Luscombe NM, Ule J: iCLIP reveals the function of hnRNP particles in splicing at individual nucleotide resolution. Nat Struct Mol Biol 2010, 17:909-915.

3. Licatalosi DD, Mele A, Fak JJ, Ule J, Kayikci M, Chi SW, Clark TA, Schweitzer AC, Blume JE, Wang X, Darnell JC, Darnell RB: HITS-CLIP yields genome-wide insights into brain alternative RNA processing. Nature 2008, 456:464-469.

4. Wang Z, Kayikci M, Briese M, Zarnack K: iCLIP predicts the dual splicing effects of TIA-RNA interactions. PLoS Biol 2010, 8:e1000530.

5. Granneman S, Kudla G, Petfalski E, Tollervey D: Identification of protein binding sites on U3 snoRNA and pre-rRNA by UV cross-linking and high-throughput analysis of cDNAs. Proc Natl Acad Sci U S A 2009, 106:9613-9618.

6. Jamonnak N, Creamer TJ, Darby MM, Schaughency P, Wheelan SJ, Corden JL: Yeast Nrd1, Nab3, and Sen 1 transcriptome-wide binding maps suggest multiple roles in post-transcriptional RNA processing. RNA 2011, 17:2011-2025

7. Wang Z, Tollervey J, Briese M, Turner D, Ule J: CLIP: construction of CDNA libraries for high-throughput sequencing from RNAs cross-linked to proteins in vivo. Methods 2009, 48:287-293.

8. Corcoran DL, Georgiev S, Mukherjee N, Gottwein E, Skalsky RL, Keene JD, Ohler U: PARalyzer: definition of RNA binding sites from PAR-CLIP short-read sequence data. Genome Biol 2011, 12:R79.

9. Althammer S, González-Vallinas J, Ballaré C, Beato M, Eyras E: Pyicos: a versatile toolkit for the analysis of high-throughput sequencing data. Bioinformatics 2011, 27:3333-3340.

10. Sievers CC, Schlumpf TT, Sawarkar RR, Comoglio FF, Paro RR: Mixture models and wavelet transforms reveal high confidence RNA-protein interaction sites in MOV10 PAR-CLIP data. Nucleic Acids Res 2012, 40:e160-e160.

11. Khorshid M, Rodak C, Zavolan M: CLIPZ: a database and analysis environment for experimentally determined binding sites of RNA-binding proteins. Nucleic Acids Res 2011, 39:D245-D252.

12. Vasiljeva $L$, Buratowski S: Nrd1 interacts with the nuclear exosome for $3^{\prime}$ processing of RNA polymerase II transcripts. Mol Cell 2006, 21:239-248.

13. Steinmetz EJ, Conrad NK, Brow DA, Corden JL: RNA-binding protein Nrd1 directs poly(A)-independent 3'-end formation of RNA polymerase II transcripts. Nature 2001, 413:327-331

14. Hobor F, Pergoli R, Kubicek K, Hrossova D, Bacikova V, Zimmermann M Pasulka J, Hofr C, Vanacova S, Stefl R: Recognition of transcription termination signal by the nuclear polyadenylated RNA-binding (NAB) 3 protein. J Biol Chem 2011, 286:3645-3657.

15. Carroll KL, Pradhan DA, Granek JA, Clarke ND, Corden JL: Identification of cis elements directing termination of yeast nonpolyadenylated snoRNA transcripts. Mol Cell Biol 2004, 24:6241-6252

16. Carroll KL, Ghirlando R, Ames JM, Corden JL: Interaction of yeast RNA-binding proteins $\mathrm{Nrd} 1$ and Nab3 with RNA polymerase II terminator elements. RNA 2007, 13:361-373.

17. Lunde BM, Hörner M, Meinhart A: Structural insights into cis element recognition of non-polyadenylated RNAs by the Nab3-RRM. Nucleic Acids Res 2011, 39:337-346.

18. Hogan DJ, Riordan DP, Gerber AP, Herschlag D, Brown PO: Diverse RNAbinding proteins interact with functionally related sets of RNAs, suggesting an extensive regulatory system. PLoS Biol 2008, 6:e255.

19. Thiebaut M, Kisseleva-Romanova E, Rougemaille M, Boulay J, Libri D: Transcription termination and nuclear degradation of cryptic unstable transcripts: a role for the nrd1-nab3 pathway in genome surveillance. Mol Cell 2006, 23:853-864.

20. Kuehner JN, Pearson EL, Moore C: Unravelling the means to an end: RNA polymerase II transcription termination. Nat Rev Mol Cell Biol 2011, 12:283-294.

21. LaCava J, Houseley J, Saveanu C, Petfalski E, Thompson E, Jacquier A Tollervey D: RNA degradation by the exosome is promoted by a nuclear polyadenylation complex. Cell 2005, 121:713-724.

22. Wyers F, Rougemaille M, Badis G, Rousselle JC, Dufour ME, Boulay J, Regnault B, Devaux F, Namane A, Séraphin B, Libri D, Jacquier A: Cryptic pol II transcripts are degraded by a nuclear quality control pathway involving a new poly(A) polymerase. Cell 2005, 121:725-737.

23. Grzechnik P, Kufel J: Polyadenylation linked to transcription termination directs the processing of snoRNA precursors in yeast. Mol Cell 2008 32:247-258

24. Wlotzka W, Kudla G, Granneman S, Tollervey D: The nuclear RNA polymerase II surveillance system targets polymerase III transcripts. EMBO J 2011, 30:1790-1803.

25. Arigo JT, Carroll KL, Ames JM, Corden JL: Regulation of yeast NRD1 expression by premature transcription termination. Mol Cell 2006, 21:641-651 
26. Kim M, Vasiljeva L, Rando OJ, Zhelkovsky A, Moore C, Buratowski S: Distinct pathways for snoRNA and mRNA termination. Mol Cell 2006, 24:723-734.

27. Gudipati RK, Villa T, Boulay J, Libri D: Phosphorylation of the RNA polymerase II C-terminal domain dictates transcription termination choice. Nat Struct Mol Biol 2008, 15:786-794.

28. Vasiljeva L, Kim M, Mutschler H, Buratowski S, Meinhart A: The Nrd1-Nab3-Sen1 termination complex interacts with the Ser5-phosphorylated RNA polymerase II C-terminal domain. Nat Struct Mol Biol 2008, 15:795-804.

29. Creamer TJ, Darby MM, Jamonnak N, Schaughency P, Hao H, Wheelan SJ, Corden JL: Transcriptome-Wide Binding Sites for Components of the Saccharomyces cerevisiae Non-Poly(A) Termination Pathway: Nrd1, Nab3, and Sen1. PLoS Genet 2011, 7:e1002329.

30. Darby MM, Serebreni L, Pan X, Boeke JD, Corden JL: The S. cerevisiae Nrd1-Nab3 Transcription Termination Pathway Acts in Opposition to Ras Signaling and Mediates Response to Nutrient Depletion. Mol Cell Biol 2012, 32:1762-1775.

31. Ciais $D$, Bohnsack MT, Tollervey D: The mRNA encoding the yeast ARE-binding protein Cth2 is generated by a novel 3' processing pathway. Nucleic Acids Res 2008, 36:3075-3084.

32. Rondón AG, Mischo HE, Kawauchi J, Proudfoot NJ: Fail-safe transcriptional termination for protein-coding genes in S. cerevisiae. Mol Cell 2009, 36:88-98.

33. Gudipati RK, Xu Z, Lebreton A, Séraphin B, Steinmetz LM, Jacquier A, Libri D: Extensive degradation of RNA Precursors by the Exosome in Wild-Type Cells. Mol Cell 2012, 48:409-421.

34. pyCRAC command line tools. [https://bitbucket.org/sgrann/pycrac]

35. Porrua O, Hobor F, Boulay J, Kubicek K, D'Aubenton-Carafa Y, Gudipati RK, Stefl R, Libri D: In vivo SELEX reveals novel sequence and structural determinants of Nrd1-Nab3-Sen1-dependent transcription termination. EMBO J 2012, 31:3935-3948.

36. Noël J-F, Larose S, Abou Elela S, Wellinger RJ: Budding yeast telomerase RNA transcription termination is dictated by the Nrd1/Nab3 non-coding RNA termination pathway. Nucleic Acids Res 2012, 40:5625-5636.

37. Kim H, Erickson B, Luo W, Seward D, Graber JH, Pollock DD, Megee PC, Bentley DL: Gene-specific RNA polymerase II phosphorylation and the CTD code. Nat Struct Mol Biol 2010, 17:1279-1286.

38. Neil H, Malabat C, D'Aubenton-Carafa Y, Xu Z, Steinmetz LM, Jacquier A: Widespread bidirectional promoters are the major source of cryptic transcripts in yeast. Nature 2009, 457:1038-1042.

39. Xu Z, Wei W, Gagneur J, Perocchi F, Clauder-Münster S, Camblong J, Guffanti E, Stutz F, Huber W, Steinmetz LM: Bidirectional promoters generate pervasive transcription in yeast. Nature 2009, 457:1033-1037.

40. Nagalakshmi U, Wang Z, Waern K, Shou C, Raha D, Gerstein M, Snyder M: The transcriptional landscape of the yeast genome defined by RNA sequencing. Science 2008, 320:1344-1349.

41. Ozsolak F, Kapranov P, Foissac S, Kim SW, Fishilevich E, Monaghan AP, John B, Milos PM: Comprehensive polyadenylation site maps in yeast and human reveal pervasive alternative polyadenylation. Cell 2010, 143:1018-1029.

42. Kuehner JN, Brow DA: Regulation of a eukaryotic gene by GTPdependent start site selection and transcription attenuation. Mol Cell 2008, 31:201-211.

43. Thiebaut M, Colin J, Neil H, Jacquier A, Séraphin B, Lacroute F, Libri D: Futile cycle of transcription initiation and termination modulates the response to nucleotide shortage in S. cerevisiae. Mol Cell 2008, 31:671-682.

44. Jenks MH, O'Rourke TW, Reines D: Properties of an intergenic terminator and start site switch that regulate IMD2 transcription in yeast. Mol Cell Biol 2008, 28:3883-3893.

45. Kopcewicz KA, O'Rourke TW, Reines D: Metabolic regulation of IMD2 transcription and an unusual DNA element that generates short transcripts. Mol Cell Biol 2007, 27:2821-2829.

46. van Dijk EL, Chen CL, d'Aubenton-Carafa Y, Gourvennec S, Kwapisz M, Roche V, Bertrand C, Silvain M, Legoix-Né P, Loeillet S, Nicolas A, Thermes C, Morillon A: XUTs are a class of Xrn1-sensitive antisense regulatory non-coding RNA in yeast. Nature 2011, 475:114-117.

47. Hess DC, Myers CL, Huttenhower C, Hibbs MA, Hayes AP, Paw J, Clore JJ, Mendoza RM, Luis BS, Nislow C, Giaever G, Costanzo M, Troyanskaya OG, Caudy AA: Computationally driven, quantitative experiments discover genes required for mitochondrial biogenesis. PLOS Genet 2009, 5:e1000407.

48. Pelechano V, Wei W, Steinmetz LM: Extensive transcriptional heterogeneity revealed by isoform profiling. Nature 2013, 497:127-131.

49. pyCRAC for Galaxy. [http://toolshed.g2.bx.psu.edu/view/swebb/pycrac]
50. FASTX-Toolkit. [http://hannonlab.cshl.edu/fastx_toolkit/]

51. Novoalign. [http://www.novocraft.com/main/index.php]

52. Saccharomyces Genome Database. [http://www.yeastgenome.org]

53. Cluster. [http://bonsai.hgc.jp/ mdehoon/software/cluster/index.html]

54. Java TreeView. [http://jtreeview.sourceforge.net]

55. Tollervey D, Mattaj IW: Fungal small nuclear ribonucleoproteins share properties with plant and vertebrate U-snRNPs. EMBO J 1987, 6:469-476.

doi:10.1186/gb-2014-15-1-r8

Cite this article as: Webb et al:: PAR-CLIP data indicate that Nrd1-Nab3dependent transcription termination regulates expression of hundreds of protein coding genes in yeast. Genome Biology 2014 15:R8.

\section{Submit your next manuscript to BioMed Central and take full advantage of:}

- Convenient online submission

- Thorough peer review

- No space constraints or color figure charges

- Immediate publication on acceptance

- Inclusion in PubMed, CAS, Scopus and Google Scholar

- Research which is freely available for redistribution

Submit your manuscript at www.biomedcentral.com/submit
C Biomed Central 